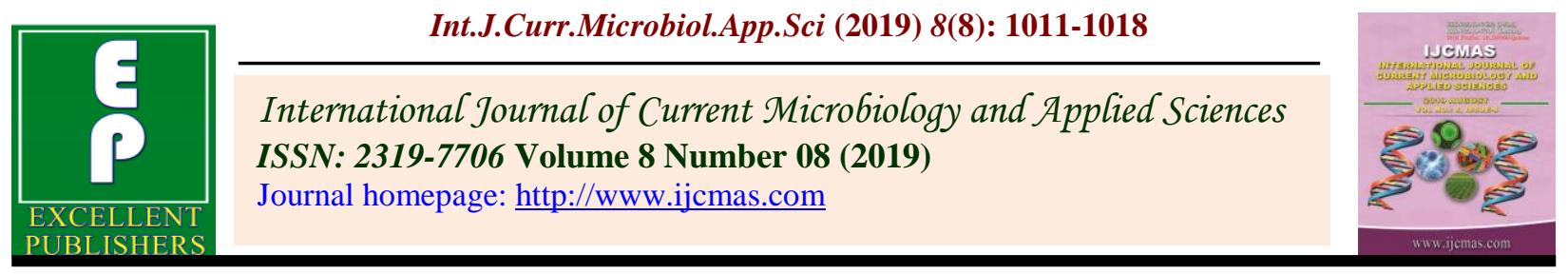

Original Research Article

https://doi.org/10.20546/ijcmas.2019.808.117

\title{
Character Association and Path Analysis Studies in Vegetable Soybean (Glycine max L.)
}

\author{
N. Shilpashree ${ }^{1 *}$, S. Nirmala Devi ${ }^{2}$, P. Anitha ${ }^{2}$ and P. Indira ${ }^{2}$ \\ ${ }^{1}$ College of Horticulture, Vellanikkara, KAU, Thrissur, India \\ ${ }^{2}$ Department of Vegetable Science, Kerala Agricultural University, Thrissur-650562, India \\ *Corresponding author
}

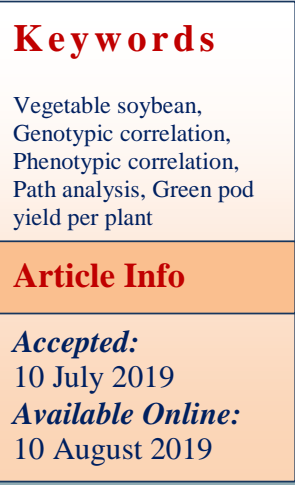

\section{A B S T R A C T}

The investigations on Character association and path analysis studies in vegetable soybean (Glycine max L.) revealed that green pod yield per plant was significantly and positively correlated with number of pods per plant, number of harvests, vitamin $\mathrm{C}$ content and calcium content at genotypic and phenotypic correlation levels. Hence direct selection for these traits will lead to the selection of high yielding genotypes of vegetable soybean. Path analysis of data revealed that number of pods per plant showed highest direct positive effect on green pod yield per plant followed by pod weight, days to first harvest and pod length. Plant height, days to vegetable maturity and pod width had direct negative effect on green pod yield per plant at genotypic and phenotypic levels.

\section{Introduction}

Vegetable soybean Glycine max (L.) Merrill is characterized by their large seed size, pleasing aroma, sweet taste, bright green colour, light hilum and soft texture with better digestibility compared to grain soybean. When freshly harvested (tender green pods at $80 \%$ maturity stage) it serves as a good vegetable like green peas, lima beans or hyacinth beans. It has great potential to enhance the nutritional value of human diet because of its high dietary fibre content, health promoting phyto-chemicals, proteins, unsaturated fats, phosphorus, thiamine and riboflavin(Carson et al., 2012;
Ponnusha et al., 2011). Among the quantitative characters, yield is a complex character and is dependent on yield components. Therefore, the knowledge on correlation between yield and its contributing characters are essential to formulate guidelines for selection of desirable types. Partitioning of total correlation into direct and indirect effect by path coefficient analysis helps in making the selection more effective. Keeping this in view, the present investigation was carried out in the Department of Vegetable Science, College of Horticulture, Vellanikkarato study the correlation between yield and its component characters and to partition total 
correlation into direct and indirect effect in 28 genotypes of vegetable soybean (Table 1).

\section{Materials and Methods}

The experiment was laid out with 28 genotypes in randomized block design with three replications during October-December, 2017 at spacing of $45 \mathrm{~cm} \mathrm{x} 45 \mathrm{~cm}$. There were twenty plants per plot per genotype. The cultural practises were followed as per the Package of Practises Recommendations (2016) of Kerala Agricultural University.

Five plants were selected at random from each plot to record observations on plant height, days to $50 \%$ flowering, days to first harvest, days to vegetable maturity, pod length, pod width, pod yield per plant, number of pods per plant, pod weight, number of harvests and quality parameters viz. starch, carbohydrate, protein, crude fibre, vitamin C, polyphenols, phosphorous, calcium and iron and the average was calculated for further statistical analysis.

\section{Results and Discussion}

\section{Character association}

Character association analysis gives an idea about relationship among the various characters and determines the component characters based on which selection for genetic improvement in pod yield can be done. The yield components may be independent or interlinkedin their nature. Correlation coefficient (Table 2 and 3) is used to find out the degree and direction of relationship between two or more characters.

In this experiment green pod yield per plant is highly significant and positively correlated with number of pods per plant $(\mathrm{rG}=0.2612)$, vitamin $\mathrm{C}(\mathrm{rG}=0.249)$ and calcium content $(\mathrm{rG}=0.27125)$ and number of harvest $(\mathrm{rG}=$ 0.256). It was also positively but non significantly correlated with plant height (0.1433), days to $50 \%$ flowering (0.1539), days to first harvest (0.1642), pod width (0.0951), pod weight $(0.2065)$, crude fibre (0.1741) and polyphenol content (0.147). Positive and significant correlation was found for days to $50 \%$ flowering with days to first harvest $(\mathrm{rG}=0.952)$, days to vegetable maturity $(\mathrm{rG}=0.2838)$ and phosphorous $(\mathrm{rG}=$ 0.342 ) but it was significant and negatively correlated with number of harvests $(\mathrm{rG}=$ 0.493), starch content ( $\mathrm{rG}=-0.746)$, carbohydrate content $(\mathrm{rG}=-0.658)$ and polyphenol content $(\mathrm{rG}=-0.2533)$.

Plant height had positive and significant association with number of pods per plant $(\mathrm{rG}$ $=0.313)$ and pod weight $(\mathrm{rG}=0.429)$. Positive and significant correlation was found for days to $50 \%$ flowering with days to first harvest $(\mathrm{rG}=0.952)$, days to vegetable maturity $(\mathrm{rG}=0.2838)$ and phosphorous $(\mathrm{rG}=$ 0.342 ) but it was significant and negatively correlated with number of harvests $(\mathrm{rG}=$ 0.493), starch content ( $\mathrm{rG}=-0.746)$, carbohydrate content $(\mathrm{rG}=-0.658)$ and polyphenol content $(\mathrm{rG}=-0.2533)$.

Table.1 List of the genotypes used in the experiment

\begin{tabular}{|l|l|}
\hline Genotypes & Source \\
\hline GM-1, GM-2, GM-3, GM-4, GM-5, GM-6, GM-7, GM-8, & \\
GM-9, GM-10, GM-11, GM-12, GM-13, GM-14, GM-15, & IIHR, Bengaluru \\
GM-16, GM-17, GM-18, GM-19, GM-20, GM-21, GM- & \\
\hline 22, GM-23, GM-24, GM-25, GM-26, GM-27, GM-28 & \\
\hline
\end{tabular}


Table.2 Phenotypic correlation coefficients among morphological and quality parameters in vegetable soybean

\begin{tabular}{|c|c|c|c|c|c|c|c|c|c|c|c|c|c|c|c|c|c|c|c|}
\hline & 1 & 2 & 3 & 4 & 5 & 6 & 7 & 8 & 9 & 10 & 11 & $' 12$ & 13 & 14 & 15 & 16 & 17 & 18 & 19 \\
\hline 1 & $1 * *$ & & & & & & & & & & & & & & & & & & \\
\hline 2 & $0.013^{*}$ & $1 * *$ & & & & & & & & & & & & & & & & & \\
\hline 3 & 0.050 & $0.875^{* *}$ & $1 * *$ & & & & & & & & & & & & & & & & \\
\hline 4 & -0.086 & $0.233^{*}$ & $0.305^{* *}$ & $1 * *$ & & & & & & & & & & & & & & & \\
\hline 5 & $-0.235^{*}$ & -0.014 & -0.153 & -0.207 & $1 * *$ & & & & & & & & & & & & & & \\
\hline 6 & $-0.42 * *$ & -0.160 & -0.103 & 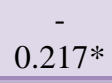 & $0.502 * *$ & $1 * *$ & & & & & & & & & & & & & \\
\hline 7 & $0.279 *$ & 0.061 & 0.004 & -0.185 & -0.077 & -0.100 & $1 * *$ & & & & & & & & & & & & \\
\hline 8 & $0.413 * *$ & 0.073 & 0.028 & $\overline{-}^{-}$ & -0.129 & 0.024 & $0.729 * *$ & $1 * *$ & & & & & & & & & & & \\
\hline 9 & $-0.29 * *$ & $-0.45 * *$ & $-0.42 * *$ & 0.063 & 0.175 & 0.037 & $-0.253 *$ & $-0.56 * *$ & $1 * *$ & & & & & & & & & & \\
\hline 10 & -0.100 & $-0.69 * *$ & $-0.71 * *$ & $-0.25^{*}$ & 0.220 & 0.188 & -0.182 & -0.076 & $0.287 * *$ & $1 * *$ & & & & & & & & & \\
\hline 11 & -0.137 & $-0.61 * *$ & $-0.60 * *$ & ${ }^{-}-{ }^{-} 0^{* *}$ & 0.026 & $0.341 * *$ & -0.136 & 0.099 & $0.274^{*}$ & $0.577 * *$ & $1 * *$ & & & & & & & & \\
\hline 12 & 0.194 & 0.013 & 0.171 & 0.107 & $-0.36^{* *}$ & -0.199 & -0.034 & 0.062 & 0.012 & -0.083 & $0.250^{*}$ & $1 * *$ & & & & & & & \\
\hline 13 & 0.054 & -0.019 & -0.039 & $\overline{-}^{-}$ & $0.297 * *$ & $0.358 * *$ & 0.026 & 0.175 & $-0.233^{*}$ & 0.487 & -0.062 & $\begin{array}{c}- \\
0.255\end{array}$ & $1 * *$ & & & & & & \\
\hline 14 & -0.156 & -0.071 & -0.138 & -0.097 & 0.065 & 0.077 & 0.127 & 0.174 & 0.144 & 0.286 & 0.30 & 0.179 & 0.146 & $1 * *$ & & & & & \\
\hline 15 & 0.117 & -0.118 & -0.022 & -0.130 & 0.127 & -0.142 & -0.154 & -0.159 & 0.016 & 0.161 & 0.033 & 0.212 & -0.137 & -0.182 & $1 * *$ & & & & \\
\hline 16 & $-0.43 * *$ & 0.138 & 0.052 & 0.030 & 0.077 & 0.358 & $0.224 *$ & $0.242 *$ & -0.040 & 0.058 & 0.172 & $\begin{array}{c}- \\
0.095\end{array}$ & $0.267 *$ & $0.488 * *$ & $\begin{array}{l}- \\
0.173\end{array}$ & $1 * *$ & & & \\
\hline 17 & -0.148 & $0.326 * *$ & $0.237 *$ & 0.094 & -0.087 & -0.083 & 0.031 & 0.205 & $-0.38 * *$ & $-0.40 * *$ & -0.114 & 0.009 & -0.190 & $-0.076 *$ & $\begin{array}{c}- \\
0.111\end{array}$ & -0.054 & $1 * *$ & & \\
\hline 18 & -0.055 & $-0.233^{*}$ & $-0.257 *$ & $0.37^{-} * *$ & 0.064 & 0.117 & $0.317 * *$ & $0.294 * *$ & -0.068 & $0.368 * *$ & 0.200 & $\begin{array}{c}- \\
0.109\end{array}$ & $0.406 * *$ & $0.454 * *$ & 0.088 & $0.429 * *$ & ${ }^{-}-{ }^{-} 39^{*}$ & $1 * *$ & \\
\hline 19 & 0.143 & 0.148 & 0.156 & -0.106 & -0.079 & 0.093 & $0.230 *$ & 0.202 & $0.256^{*}$ & -0.206 & -0.100 & 0.063 & 0.173 & $0.243^{*}$ & $\begin{array}{c}- \\
0.170\end{array}$ & $0.270 *$ & $0 . \overline{246 *}$ & 0.145 & $1 * *$ \\
\hline
\end{tabular}

*Significant at $5 \%$

$* *$ Significant at $1 \%$

\begin{tabular}{|l|l|l|}
\hline 1. Plant height & 6. Pod width & 11. Carbohydrate \\
\hline 2. Days to $\mathbf{5 0 \%}$ flowering & 7. Number of pods per plant & 12. Protein \\
\hline 3. Days to first harvest & 8. Pod weight & 13. Crude fibre \\
\hline 4. Days to vegetable maturity & 9. Number of harvests & 14. Vitamin C \\
\hline 5. Pod length & 10. Starch & 15. Iron \\
\hline
\end{tabular}


Table.3 Genotypic correlation coefficients among morphological and quality parameters in vegetable soybean

\begin{tabular}{|c|c|c|c|c|c|c|c|c|c|c|c|c|c|c|c|c|c|c|c|}
\hline & 1 & 2 & 3 & 4 & 5 & 6 & 7 & 8 & 9 & 10 & 11 & 12 & 13 & 14 & 15 & 16 & 17 & 18 & 19 \\
\hline 1 & $1 * *$ & & & & & & & & & & & & & & & & & & \\
\hline 2 & -0.0116 & $1 * *$ & & & & & & & & & & & & & & & & & \\
\hline 3 & 0.0567 & $0.952 * *$ & $1 * *$ & & & & & & & & & & & & & & & & \\
\hline 4 & -0.0936 & $0.2838 * *$ & $0.3545^{*}$ & $1 * *$ & & & & & & & & & & & & & & & \\
\hline 5 & $-{ }^{-}$ & -0.1459 & -0.1665 & $-0.227^{*}$ & $1 * *$ & & & & & & & & & & & & & & \\
\hline 6 & $-0.42 * *$ & -0.170 & -0.1094 & $-0.228^{*}$ & $0.5044 * *$ & $1 * *$ & & & & & & & & & & & & & \\
\hline 7 & $0.3125 *$ & 0.1215 & 0.0700 & $-0.271^{*}$ & -0.090 & -0.1113 & $1 * *$ & & & & & & & & & & & & \\
\hline 8 & $0.429 * *$ & 0.0909 & 0.0511 & $-0.385^{*}$ & -0.135 & 0.0258 & $0.779 * *$ & $1 * *$ & & & & & & & & & & & \\
\hline 9 & $-0.29 * *$ & $-0.49 * *$ & $-0.46^{* *}$ & 0.1096 & 0.1803 & 0.0360 & $-0.29 * *$ & $-0.61 * *$ & $1 * *$ & & & & & & & & & & \\
\hline 10 & -0.1074 & $-0.74 * *$ & $-0.78 * *$ & $-0.274^{*}$ & $0.2269^{*}$ & 0.1935 & -0.1770 & -0.0780 & $0.3169 * *$ & $1 * *$ & & & & & & & & & \\
\hline 11 & -0.1378 & $-0.65 * *$ & $-0.65 * *$ & $-0.32 * *$ & 0.0256 & $0.343^{* *}$ & -0.1490 & 0.1035 & $0.2811 * *$ & $0.6042^{*}$ & $1 * *$ & & & & & & & & \\
\hline 12 & 0.1941 & 0.0185 & 0.1841 & 0.1202 & $-0.370 * *$ & -0.2003 & -0.0329 & 0.0650 & 0.0139 & -0.0872 & $0.2510^{*}$ & $1 * *$ & & & & & & & \\
\hline 13 & 0.0543 & -0.0227 & -0.0441 & $-0.36 * *$ & $0.2987 * *$ & $0.359^{* *}$ & 0.0316 & 0.1792 & $-0.2405^{*}$ & 0.1532 & -0.0621 & $\stackrel{-}{-}$ & $1 * *$ & & & & & & \\
\hline 14 & -0.1639 & -0.0854 & -0.1514 & -0.1203 & 0.06799 & 0.0735 & 0.1465 & 0.1877 & 0.1565 & 0.2973* & $0.3093 * *$ & 0.1840 & 0.15035 & $1 * *$ & & & & & \\
\hline 15 & 0.1186 & -0.1258 & -0.0197 & -0.1398 & 0.1274 & -0.1425 & -0.17985 & -0.1670 & 0.01312 & 0.1698 & 0.03289 & 0.2129 & -0.1378 & $\begin{array}{c}- \\
0.18775\end{array}$ & $1 * *$ & & & & \\
\hline 16 & $-0.43^{* *}$ & 0.14410 & 0.0578 & 0.03904 & 0.0773 & $0.3608 * *$ & $0.2551 *$ & $0.2472 *$ & -0.0485 & 0.0635 & 0.1734 & -0.0960 & $0.2684 *$ & $0.499 * *$ & $\overline{-}-17443$ & $1 * *$ & & & \\
\hline 17 & -0.1489 & $0.3422 *$ & $0.2560 *$ & 0.10209 & -0.0881 & -0.0834 & 0.03518 & 0.21098 & $-0.404 * *$ & $-0.41 * *$ & -0.1147 & 0.00964 & -0.1907 & -0.0778 & -0.1116 & -0.0548 & $1 * *$ & & \\
\hline 18 & -0.0547 & $-0.2533^{*}$ & $-0.28 * *$ & $-0.40 * *$ & 0.6434 & 0.1180 & $0.3704 * *$ & $0.30747 *$ & -0.0793 & $0.388^{* *}$ & 0.20198 & $\begin{array}{c}- \\
0.11098\end{array}$ & $0.410 * *$ & $0.467 * *$ & 0.0897 & $0.433^{* *}$ & $\begin{array}{c}- \\
0.2409 *\end{array}$ & $1 * *$ & \\
\hline 19 & 0.1433 & 0.1539 & 0.1642 & -0.1088 & -0.07891 & 0.09513 & 0.2612 & 0.2065 & $0.2709 *$ & -0.2142 & -0.10186 & -0.0636 & 0.1741 & $0.2492^{*}$ & -0.1717 & $0.2712^{*}$ & $-{ }^{-}$ & 0.14668 & $1 * *$ \\
\hline
\end{tabular}

*Significant at $5 \%$

$* *$ Significant at $1 \%$

\begin{tabular}{|l|l|l|}
\hline 1. Plant height & 6. Pod width & 11. Carbohydrate \\
\hline 2. Days to $\mathbf{5 0 \%}$ flowering & 7. Number of pods per plant & 12. Protein \\
\hline 3. Days to first harvest & 8. Pod weight & 13. Crude fibre \\
\hline 4. Days to vegetable maturity & 9. Number of harvests & 14. Vitamin C \\
\hline 5. Pod length & 10. Starch & 15. Iron \\
\hline
\end{tabular}


Table.4 Path coefficient analysis for pod yield and its components in vegetable soybean

\begin{tabular}{|c|c|c|c|c|c|c|c|c|c|}
\hline \multirow{2}{*}{ Plant height } & $\begin{array}{l}\text { Plant } \\
\text { height }\end{array}$ & $\begin{array}{l}\text { Days to } 50 \\
\text { \% flowering }\end{array}$ & $\begin{array}{l}\text { Days to } \\
\text { first } \\
\text { harvest }\end{array}$ & $\begin{array}{c}\text { Days to } \\
\text { vegetable } \\
\text { maturity }\end{array}$ & Pod length & $\begin{array}{c}\text { Pod } \\
\text { width }\end{array}$ & $\begin{array}{l}\text { Number of } \\
\text { pods per } \\
\text { plant }\end{array}$ & $\begin{array}{c}\text { Pod } \\
\text { weight }\end{array}$ & $\mathbf{r G}$ \\
\hline & -0.115 & -0.0004 & 0.0106 & 0.0013 & -0.024 & 0.099 & 0.4737 & -0.1322 & 0.143 \\
\hline $\begin{array}{l}\text { Days to } 50 \% \\
\text { flowering }\end{array}$ & 0.0013 & 0.0387 & 0.1788 & -0.0041 & -0.0151 & 0.0399 & 0.10042 & -0.2175 & 0.157 \\
\hline Days to harvest & 0.0065 & 0.0370 & 0.1874 & -0.0051 & -0.0173 & 0.0255 & 0.0567 & -0.2067 & 0.164 \\
\hline $\begin{array}{l}\text { Days to } \\
\text { vegetable } \\
\text { maturity }\end{array}$ & 0.0107 & 0.0110 & 0.0660 & -0.0144 & -0.0236 & 0.0532 & -0.4250 & 0.0486 & -0.109 \\
\hline Pod length & 0.0272 & -0.0056 & -0.0312 & 0.0033 & 0.1038 & -0.1177 & -0.1498 & 0.0795 & -0.079 \\
\hline Pod width & 0.0489 & -0.0066 & -0.0205 & 0.0033 & 0.0523 & -0.233 & 0.0285 & 0.0158 & 0.095 \\
\hline $\begin{array}{l}\text { Number of pods } \\
\text { per plant }\end{array}$ & 0.0493 & 0.0035 & 0.0096 & 0.0055 & -0.0141 & -0.006 & 1.1017 & -0.2706 & 0.261 \\
\hline \multirow[t]{2}{*}{ Pod weight } & 0.0344 & -0.0191 & -0.0879 & -0.0016 & 0.0187 & -0.0084 & -0.6763 & 0.4408 & 0.206 \\
\hline & $s$ direct eff & *Signific & cant at $5 \%$ & nificant at $1 \%$ & rG- Genotypic c & lation with & yield per plant & Residual $=0$ & \\
\hline \multicolumn{2}{|c|}{ 1. Plant height } & & 6. Pod width & & & & & & \\
\hline \multicolumn{3}{|c|}{ 2. Days to $50 \%$ flowering } & 7. Number of & er plant & & & & & \\
\hline \multicolumn{3}{|c|}{ 3. Days to first harvest } & 8. Pod weight & & & & & & \\
\hline \multicolumn{3}{|c|}{$\begin{array}{l}\text { 4. Days to vegetable maturity } \\
\text { 5. Pod length }\end{array}$} & & & & & & & \\
\hline
\end{tabular}


Kumar et al., (2004); Mallik et al., (2007); Bello et al., (2012); Li et al., (2013); Kumar et al., (2015); Ramya and Mummigatti (2015), Basavaraja et al., (2005), Sciarappa et al., (2007) and Poornima et al., (2014) also reported similar results in vegetable soybean. Protein content was negatively and significantly correlated with pod length, crude fibre and carbohydrate content. Similar results were observed by Sharma et al., (2014), Salmani et al., (2012) and Sharma et al., (2013) in vegetable soybean and Sharma et al., (2016).

\section{Path analysis}

Path coefficient analysis (Table 4) is an important tool for partitioning the correlation coefficients to direct and indirect effects of independent variables on a dependent variable. When more variables are included in correlation studies, the indirect association becomes more complex. Under such circumstances, path coefficient analysis provides an effective means of a critical examination of specific forces action to produce a given correlated and measure the relative importance of each factor. Positive direct effect on pod yield per plant was shown by plant height (0.20), days to first harvest (3.04), number of pods per plant (1.391), pod weight (1.42), number of harvests (0.39), iron content (0.54), calcium content (0.24), phosphorous content (0.16).The characters which showed negative direct effect on pod yield per plant were days to $50 \%$ flowering ($2.10)$, days to vegetable maturity $(-0.57)$, pod length (-0.147), pod width $(-0.397)$, starch content (-0.327), carbohydrate content ($0.58)$, protein content $(-0.108)$, crude fibre content (-0.148), vitamin C (-0.31) and polyphenol content (-0.75).Similar observations were earlier reported by Joshi and Mehra (1984), Roy et al., (2006); Bhushan et al., (2007); Mallik et al., (2007) in soybean; Golani et al., (2007); Mishra et al.,
(2009) in French bean; Patil et al., (2011); Singh et al., (2011); Bello et al., (2012); Pal and Singh (2012); Mehra and Singh (2012);Li et al., (2013).

It is concluded that green pod yield per plant was positively and significantly correlated with number of pods per plant, number of harvests, vitamin $\mathrm{C}$ and calcium content. Days to first harvest showed high direct positive effect on green pod yield per plant followed by pod weight, number of pods per plant, number of harvests and plant height. Therefore these characters should be considered in selection criteria for increasing green pod yield per plant.

\section{References}

Basavaraja, G. T., Naidu, G. K., and Salimath, P. M. 2005. Evaluation of vegetable soybean genotypes for yield and component traits. Karnataka. J. Agric. Sci. 18(1): 27-31.

Bello, L. L., Shaahu, A., and Vange, T. 2012. Studies on relationship between seed yield and yield components in soybean (Glycine $\max$ L. Merrill). Electr. J. Plant Breed. 3(4): 1012-1017.

Bhushan, K. B., Singh, B. P., Dubey, R. K. and Ram, H. H., 2007. Correlation analysis for seed yield in French bean (Phaseolus vulgaris). Pantnagar J. Res., 5(1): 104 -106.

Carson, L. C., Freeman, H. J., Zhou, K. and Welbaum, G. E., 2012, Antioxidant Capacity and Isoflavone Content in Seeds of Five Edamame (Glycine max L. Merrill) Cultivars. Proc. Fla.State Hort. Soc., 125: 169-173.

Czaikoski, K., Santos, R. L., Mandarino, G. J., Carrão-Panizzic, C. M., DaSilva, J. B. and Elza, I. I., 2013, Canning of vegetable-type soybean in acidified brine: Effect of the addition of sucrose and pasteurization time on color and 
other characteristics. Industrial Crops and Products, 45: 472-476.

Golani, I. J., Naliyadhara, M. V., Mehta, D. R., Purohit and Padya, H. M., 2006, Genetic divergence in dolichos bean (Lablab purpureus L.). Legume Res., 29(4): 286-288.

Joshi, B. D. and Mehra, K. L., 1984, Path analysis of productivity in French bean. Prog. Hort., 16(1-2): 78-84.

KAU [Kerala Agriculture University]. 2016. Package of Practices Recommendation: Crops $\left(15^{\text {th }}\right.$ Ed.). Kerala Agricultural University, Thrissur, 200p

Kumar, A., Pandey, A., Aochen, C., and Pattanayak, A. 2015. Evaluation of Genetic Diversity and Interrelationships of Agro-Morphological Characters in Soybean (Glycine max) Genotypes. Proc. Nat. Acad. Sci. India Sect. B. Biol. Sci. 85(2): 397- 405.

Kumar, M. K. V., 2004, Physiological basis of yield variation in french bean (Phaseolus vulgaris L.) genotypes $M$. Sc. (Agri.) Thesis, Univ. Agric. Sci., Dharwad.

Li, Y. S., Du, M., Zhang, Q. Y., Hashemi, M., Liu, X. B., and Hebert, S. J. 2013. Correlation and path coefficient analysis for yield components of vegetable soybean in northeast china. Legume Res. 36: 284-288.

Malik, M. F., Asraf, M., Qureshi, A. S., and Ghafoor, A. 2007. Assessment of genetic variability, correlation and path analyses for yield and its components in soybean. Pak. J. Bot. 39(2): 405-413.

Pal, A. K. and Singh, S., 2012, Correlation and path analysis in garden pea (Pisum sativum $\mathrm{L}$ var. Hortense). The Asian $J$. Hort., 7(2): 569-573.

Patil, S. S., Naik, M. R., Patil, P. P., and Shinde, D. A. 2011. Genetic variability, correlation and path analysis in soybean. Legume Res. 34(1): 36-40.

Ponnusha, B. S., Pasupathi, P., Boopathi, S.,
Sathiyamoorthy, S. and Rajaram, V., 2011, A complete evaluation of the antioxidant and antimicrobial potential of Glycine max. Int. J. Curr. Sci.Res., 1(2): 6-12.

Poornima, R., Koti, R. V. and Nair, R. N., 2014, Physiological basis of yield variation in vegetable soybean and organoleptic test for acceptance. Plant Archives, 14: 51-54.

Ramya, V. and Mummigatti, U. V. 2015. Characterization of vegetable soybean genotypes for phenological, physiological and yield attributing traits. Karnataka J. Agric. Sci. 28(4): 500-503.

Roy, S. K., Md. Karim, A., Islam, A., Nasimul, B. M., Khaleque, M. A. M. and Hidaka, T., 2006. Relationship between yield and its component characters of bush bean (Phaseolus vulgaris L.). South Specific Studies, 27 (1): 13-23.

Salmani, Z., Vijayalakshmi and Sajjan, J. T., 2012. Screening of selected vegetable soybean genotypes for nutrient and antinutrient factors. J. Dairying, Foods \& H.S., 31(2): 142-145.

Sciarappa, W. J., Hunsberger, L. K., Shen, D., Qing-Li Wu, Simon, J. and Hulme, B., 2007. Evaluation of edamame cultivars in New Jersey and Maryland. Fruits, Vegetables and Nursery crops. pp. 223227.

Sharma, B. K., Kushwah, S. S., Verma, K. S. and Singh, O. P., 2013, Studies on french bean (Phaseolus vulgaris L.) varieties under different $\mathrm{N}, \mathrm{P}, \mathrm{K}$ and $\mathrm{S}$ levels for growth, yield and economics. J. Hort. Sci., 8(2): 268-270.

Sharma, D. P., Dehariya, N. K., and Tiwari, A. 2014. Genetic variability, correlation and path coefficient analysis of dolichos bean genotypes (Lablab purpureus L.) Int. J. Basic App. Agric. Res. 12(2): 193-199. 
Sharma, R. K., Kumar, S., Maji, S., Pandey, V. J., Singh, S., Dubey A. K. and Kumar, P., 2016. Genetic variability, heritability, correlation coefficient and path analysis in French bean (Phaseolus vulgaris L.). The Bioscan, 11(2): 11531153.

Shinde, S. S. and Dumbre, A. D., 2001. Correlation and path coefficient analysis in French bean. J. Maharashtra Agric. Univ., 26(1): 48-49.
Singh, Pramod K., Rai, N., Lal, Hira, Bhardwaj, D.R. Singh, Rashmi. and Prakash A., 2011. Correlation, path and cluster analysis in hyacinth bean (Lablab purpureusL. Sweet). J. Agri. Tech., 7(4): 1117-1124.

Vaid, K. S., Singh, R. M. and Gupta, V. P., 1986. Inter-relationship of yield and its component characters in dry beans (Phaseolus vulgaris L.). Crop Improv., 13: 164-167.

\section{How to cite this article:}

Shilpashree, N., S. Nirmala Devi, P. Anitha and Indira, P. 2019. Character Association and Path Analysis Studies in Vegetable Soybean (Glycine max L.). Int.J.Curr.Microbiol.App.Sci. 8(08): 1011-1018. doi: https://doi.org/10.20546/ijcmas.2019.808.117 